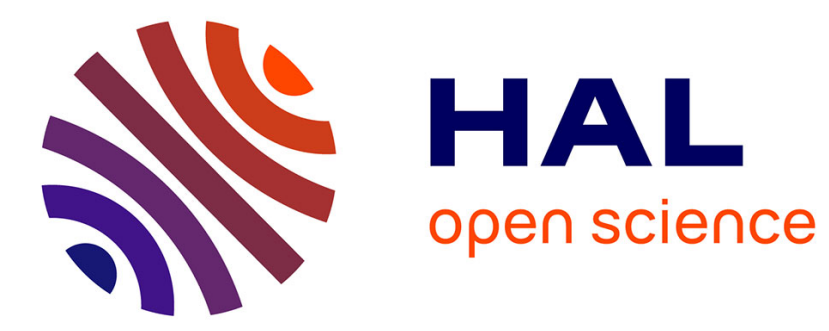

\title{
On Finite-Time Stabilization of Evolution Equations: A Homogeneous Approach
}

\author{
Andrey Polyakov, Jean-Michel Coron, Lionel Rosier
}

\section{To cite this version:}

Andrey Polyakov, Jean-Michel Coron, Lionel Rosier. On Finite-Time Stabilization of Evolution Equations: A Homogeneous Approach. IEEE Conference on Decision and Control 2016, Dec 2016, Las Vegas, United States. hal-01371089

\section{HAL Id: hal-01371089 \\ https://hal.inria.fr/hal-01371089}

Submitted on 23 Sep 2016

HAL is a multi-disciplinary open access archive for the deposit and dissemination of scientific research documents, whether they are published or not. The documents may come from teaching and research institutions in France or abroad, or from public or private research centers.
L'archive ouverte pluridisciplinaire HAL, est destinée au dépôt et à la diffusion de documents scientifiques de niveau recherche, publiés ou non, émanant des établissements d'enseignement et de recherche français ou étrangers, des laboratoires publics ou privés. 


\title{
On Finite-Time Stabilization of Evolution Equations: A Homogeneous Approach
}

\author{
Andrey Polyakov, Jean-Michel Coron, Lionel Rosier
}

\begin{abstract}
Generalized monotone dilation in a Banach space is introduced. Classical theorems on existence and uniqueness of solutions of nonlinear evolution equations are revised. A universal homogeneous feedback control for a finite-time stabilization of linear evolution equation in a Hilbert space is designed using homogeneity concept. The design scheme is demonstrated for distributed finite-time control of heat and wave equations.
\end{abstract}

\section{INTRODUCTION}

The homogeneity is a sort of symmetry of an object, when it remains consistent with respect to a dilation operation [1], [2], [3], [4], [5], [6], [7], [8]. For models described by ordinary differential equations (ODEs) homogeneity is applied for control and observer design as well as for analysis of qualitative behavior of the closed-loop systems. There are a lot of controllers and observers with finite convergence time with negative homogeneous degree to the closed-loop system, since asymptotic stability in this case implies the finite-time one [1], [9], [10], [11], [12], [13], [14], [15]. For example, in [16], [10], [12], [13], [15], [17] homogeneous feedbacks are designed for fast robust stabilization of linear plants, but in [12], [18], [19], [20] homogeneous dynamic observers are developed for non-asymptotic state estimation. Finally, homogeneity allows robustness analysis to be done easily for essentially nonlinear systems [21], [22], [23].

Partial differential equations with homogeneous (with respect to uniform scaling) operator were studied in [24], [25], [26], where some regularizing effects and integrability properties of homogeneous systems have been discovered. A version of weighted homogeneity for sub-elliptic operators is considered in [5]. In [14] a generalized (geometric) homogeneity for evolution equation is introduced. It was demonstrated that all important properties of homogeneous systems like scalability of solutions, expansion of local results to global cases and finite-time stability for negative degree can also be discovered for homogeneous evolution equations in Banach spaces. Generalized homogeneity can be established for many well-known partial differential equations [14] like $\mathrm{KdV}$, Saint-Venant, heat, wave and fast diffusion equations.

The finite-time control of partial differential equations is a topic of intensive research [27], [28], [29], [30], [31]. This problem is related with analysis of controllability and null controllability of the system. This paper presents the first

This work is supported by ANR Project Finite4SoS (ANR 15-CE230007).

Andrey Polyakov is with Inria, Lille, France, (andrey.polyakov@inria.fr). Jean-Michel Coron is with Laboratoire Jacques-Louis Lions, Université Pierre et Marie Curie, Paris, France (coron@ann.jussieu.fr)

Lionel Rosier is with Centre Automatique et Systemes, Ecole des Mines de Paris, France, (lionel.rosier@mines-paristech.fr) attempts to design finite-time control based on generalized homogeneity presented in [14]. First, the problem of existence of solutions of quasi-linear systems with homogeneous non-linearity is studied. Next, a novel universal scheme of homogeneous finite-time control design for evolution equation in a Hilbert space is developed using properties of homogeneous norms of smooth monotone dilations.

Notation: $\mathbb{R}$ is the field of real numbers; $\mathbb{R}_{+}=[0,+\infty) ; \mathbf{B}$ is a real Banach space with the norm $\|\cdot\| ; \mathbf{H}$ is a real Hilbert space with the inner product $\langle\cdot, \cdot\rangle ; H^{n}(X, \mathbb{R})=W^{n, 2}(X, \mathbb{R})$ is the Sobolev space of functions $X \rightarrow \mathbb{R}$ (with $H^{0}=L^{2}$ ), where $X \subset \mathbb{R}$ is any open set.

\section{Homogeneous Dilations in Banach Spaces}

A. Dilation group

Let $\mathcal{L}(\mathbf{B})$ be the space of linear bounded operators $\mathbf{B} \rightarrow \mathbf{B}$.

Definition 1 ([14]): A map d : $\mathbb{R} \rightarrow \mathcal{L}(\boldsymbol{B})$ is called dilation in the space $\boldsymbol{B}$ if it satisfies

- the semigroup property: $\boldsymbol{d}(0)=I \in \mathcal{L}(\boldsymbol{B})$ and $\boldsymbol{d}(t+$ $s)=\boldsymbol{d}(t) \boldsymbol{d}(s)$ for $t, s \in \mathbb{R}$;

- the strong continuity property: the map $d(\cdot) u: \mathbb{R} \rightarrow \boldsymbol{B}$ is continuous for any $u \in \boldsymbol{B}$;

- the limit property: $\lim _{s \rightarrow-\infty}\|\boldsymbol{d}(s) u\|=0$ and $\lim _{s \rightarrow+\infty}\|\boldsymbol{d}(s) u\|=\infty$ uniformly on $u \in S$.

The dilation $\mathbf{d}$ is strongly continuous group [14].

Definition 2 ([14]): A nonempty set $\mathcal{D} \subseteq \boldsymbol{B}$ is said to be $\boldsymbol{d}$-homogeneous iff $\mathcal{D}$ is invariant with respect to dilation $\boldsymbol{d}: \mathbb{R} \rightarrow \mathcal{L}(\boldsymbol{B})$, i.e. $\boldsymbol{d}(s) z \in \mathcal{D}$ for $z \in \mathcal{D}$ and $s \in \mathbb{R}$.

In particular, the $\mathbf{d}$-homogeneous set $\mathcal{D}$ may be a cone in $\mathbf{B}$.

The homogeneous sphere of the radius $r$ is given by $S_{\mathbf{d}}(r)=\{u \in \mathcal{D}: \mathbf{d}(-\ln (r)) u \in S\}, \quad r>0$.

Obviously, $S_{\mathbf{d}}(1)=S \cap \mathcal{D}$. Let us denote $\|g\|_{\mathcal{L}}^{\mathcal{D}}=\sup _{u \in S_{\mathbf{d}}(1)}\|g(u)\|, \quad\lfloor g\rfloor_{\mathcal{L}}^{\mathcal{D}}=\inf _{u \in S_{\mathbf{d}}(1)}\|g(u)\|$

for $g \in \mathcal{L}(\mathbf{B})$. Obviously, $\|g\|_{\mathcal{L}}^{\mathbf{B}}$ is the canonical norm in $\mathcal{L}(\mathbf{B})$. The limit property of the dilation immediately implies that $\|\mathbf{d}(s)\|_{\mathcal{L}}^{\mathbf{B}} \rightarrow 0$ as $s \rightarrow-\infty$ and $\lfloor g\rfloor_{\mathcal{L}}^{\mathbf{B}} \rightarrow+\infty$ as $s \rightarrow+\infty$.

Homogeneous sets and spheres have some useful properties to be utilized below for analysis of evolution equation. If $\mathcal{D}$ is a nontrivial ${ }^{1} \mathbf{d}$-homogeneous set then [14]

- $\|\mathbf{d}(s)\|_{\mathcal{L}}^{\mathcal{D}} \neq 0, s \in \mathbb{R}$ and $\mathbf{d}(s) \neq I \in \mathcal{L}(\mathbf{B})$ if $s \neq 0$;

- $\lfloor\mathbf{d}(s)\rfloor_{\mathcal{L}}^{\mathcal{D}}<1$ if $s<0$ and $1<\|\mathbf{d}(s)\|_{\mathcal{L}}<\infty$ if $s>0$;

- $S_{\mathbf{d}}(1)=\mathcal{D} \cap S \neq \emptyset$ and for any $u \in \mathcal{D} \backslash\{\boldsymbol{0}\}$ there exist $u_{0} \in S_{\mathbf{d}}(1)$ and $s_{0} \in \mathbb{R}$ such that $u=d\left(s_{0}\right) u_{0}$;

- $\mathcal{D} \backslash\{\boldsymbol{0}\}=\bigcup_{r>0} S_{\mathbf{d}}(r)$ and $S_{\mathbf{d}}(r)=\mathbf{d}(\ln (r)) S_{\mathbf{d}}(1)$.

${ }^{1}$ The set $\mathcal{D}$ is non-trivial if it contains elements different from $\mathbf{0}$. 


\section{B. Generator of dilation}

The operator $G_{\mathbf{d}}: \mathcal{D}\left(G_{\mathbf{d}}\right) \subset \mathbf{B} \rightarrow \mathbf{B}$ defined as

$$
G_{\mathbf{d}} u=\lim _{s \rightarrow 0} s^{-1}(\mathbf{d}(s) u-u)
$$

is known (see, e.g. [32, Ch. 1]) as the generator of the group $\mathbf{d}(s)$ with the domain $\mathcal{D}\left(G_{\mathbf{d}}\right)=\left\{u \in \mathbf{B}: \lim _{s \rightarrow 0} s^{-1}(\mathbf{d}(s) u-\right.$ $u$ ) exists $\}$ dense in $\mathbf{B}$. Obviously, the domain $\mathcal{D}\left(G_{\mathbf{d}}\right)$ is $\mathbf{d}$ homogeneous linear subspace of $\mathbf{B}$. The generator $G_{\mathbf{d}}$ is a linear possibly unbounded operator, which satisfies the following property ([32, Ch. 1, Theorem 2.4]: for any $u \in$ $\mathcal{D}\left(G_{\mathbf{d}}\right)$ and any $s \in \mathbb{R}_{+}$one holds $\mathbf{d}(s) u \in \mathcal{D}\left(G_{\mathbf{d}}\right)$ and the function $\mathbf{d}(\cdot) u: \mathbb{R}_{+} \rightarrow \mathcal{D}\left(G_{\mathbf{d}}\right)$ is continuously differentiable

$$
\frac{d}{d s} \mathbf{d}(s) u=G_{\mathbf{d}} \mathbf{d}(s) u=\mathbf{d}(s) G_{\mathbf{d}} u .
$$

Example: If $\mathbf{B}=H^{0}(\mathbb{R}, \mathbb{R})$ then the dilation defined as $\mathbf{d}(s) u(x)=e^{s} u\left(e^{\nu s} x\right), s \in \mathbb{R}_{+}, u \in \mathbf{B}, x \in \mathbb{R}$ has the generator $\left(G_{\mathbf{d}} u\right)(x)=\nu x u^{\prime}(x)+u(x)$.

Remark 1: If the generator $G_{\mathbf{d}}$ is a bounded linear operator then the group $\mathbf{d}$ is uniformly continuous [32, Ch.1], $\|\mathbf{d}(s)\|_{\mathcal{L}}^{\mathbf{B}} \leq e^{\left\|G_{\mathbf{d}}\right\| s}$ and $\frac{d}{d s} \mathbf{d}(s)=G_{\mathbf{d}} \mathbf{d}(s)$ for any $s \in \mathbb{R}$.

\section{Monotone dilations}

In this paper we deal with so-called monotone dilations.

Definition 3: The dilation $\boldsymbol{d}$ is monotone on d-homogeneous set $\mathcal{D} \subset \boldsymbol{B}$ if it is a strong contraction for $s<0$, i.e.

$$
\|\boldsymbol{d}(s)\|_{\mathcal{L}}^{\mathcal{D}}<1, \quad \forall s<0 .
$$

It is worth stressing that monotonicity of dilation depends on the norm $\|\cdot\|$. For example, the dilation $\mathbf{d}(s)=\left(\begin{array}{cc}e^{s} & 0 \\ 0 & e^{2 s}\end{array}\right)$ is monotone with respect to $\mathbf{B}=\mathbb{R}^{2}$ equipped with the conventional Euclidean norm, but it is non-monotone in the case of the weighted norm $\|u\|_{P}=\sqrt{u^{T} P u}$ with

$$
P=\left(\begin{array}{cc}
\frac{\sqrt{3}}{2} & \frac{1}{2} \\
-\frac{1}{2} & \frac{\sqrt{3}}{2}
\end{array}\right)\left(\begin{array}{cc}
100 & 0 \\
0 & \frac{1}{100}
\end{array}\right)\left(\begin{array}{cc}
\frac{\sqrt{3}}{2} & -\frac{1}{2} \\
\frac{1}{2} & \frac{\sqrt{3}}{2}
\end{array}\right) .
$$

In the latter case, the curve $\{\mathbf{d}(s) u: s \in \mathbb{R}\}$ may cross the homogeneous sphere in two different points. One has the following proposition, which is proved in Appendix A.

Proposition 1: If the dilation $\boldsymbol{d}$ is monotone on $\boldsymbol{d}$ homogeneous set $\mathcal{D} \subset \boldsymbol{B}$ then

1) $\lfloor\boldsymbol{d}(s)\rfloor_{\mathcal{L}}^{\mathcal{D}}>1$ for $s>0$;

2) for any $u \in \mathcal{D}$ there exists a unique pair $\left(s_{0}, u_{0}\right) \in$ $\mathbb{R} \times S_{\boldsymbol{d}}(1)$ such that $u=d\left(s_{0}\right) u_{0}$;

3) the function $\|\boldsymbol{d}(\cdot) u\|: \mathbb{R} \rightarrow \mathbb{R}_{+}$(with $u \in \mathcal{D} \backslash\{\boldsymbol{0}\}$ ) is continuous and strictly monotone increasing on $\mathbb{R}$;

4) the function $\|\boldsymbol{d}(\cdot)\|_{\mathcal{L}}^{\mathcal{D}}: \mathbb{R} \rightarrow \mathbb{R}_{+}\left(\lfloor\boldsymbol{d}(\cdot)\rfloor_{\mathcal{L}}^{\mathcal{D}}: \mathbb{R} \rightarrow \mathbb{R}_{+}\right)$is lower(upper) semicontinuous and strictly increasing: $\left\lfloor\boldsymbol{d}\left(0^{-}\right)\right\rfloor_{\mathcal{L}}^{\mathcal{D}} \cdot\left\|\boldsymbol{d}\left(0^{+}\right)\right\|_{\mathcal{L}}^{\mathcal{D}}=\left\|\boldsymbol{d}\left(0^{-}\right)\right\|_{\mathcal{L}}^{\mathcal{D}}=\left\lfloor\boldsymbol{d}\left(0^{+}\right)\right\rfloor_{\mathcal{L}}^{\mathcal{D}}=1$.

\section{Homogeneous norm}

For d-homogeneous set $\mathcal{D}$ we can introduce the so-called homogeneous "norm".

Definition 4: A functional $p: \mathcal{D} \subset \boldsymbol{B} \rightarrow \mathbb{R}_{+}$is said to be $\boldsymbol{d}$-homogeneous norm on $\mathcal{D}$ if $p(u) \rightarrow 0$ as $u \rightarrow \boldsymbol{0}$ and $p(\boldsymbol{d}(s) u)=e^{s} p(u)>0$ for $u \in \mathcal{D} \backslash\{\boldsymbol{0}\}, s \in \mathbb{R}$.

In this paper we introduce the canonical homogeneous "norm" for monotone dilation $\mathbf{d}$ as follows

$$
\|u\|_{\mathbf{d}}=e^{s_{u}}, \text { where } s_{u} \in \mathbb{R}: \mathbf{d}\left(-s_{u}\right) u \in S_{\mathbf{d}}(1) .
$$

In this case the identity $\|u\|_{\mathbf{d}}=r$ implies $u \in S_{\mathbf{d}}(r), r \geq 0$.

The next obvious relation relates the norm in the Banach space $\mathbf{B}$ and its homogeneous norm:

$$
\sigma_{1}\left(\|u\|_{\mathbf{d}}\right) \leq\|u\| \leq \sigma_{2}\left(\|u\|_{\mathbf{d}}\right)
$$

where $\sigma_{1}(\cdot) \leq \frac{1}{\|\mathbf{d}(-\ln (\cdot))\|_{\mathcal{L}}^{\mathbf{B}}}$ and $\sigma_{2}(\cdot) \geq \frac{1}{[\mathbf{d}(-\ln (\cdot))\rfloor_{\mathcal{L}}^{\mathrm{B}}}$ are some continuous increasing functions.

The homogeneous "norm" $\|\cdot\|_{\mathbf{d}}$ is not a norm in the classical sense, but it defines a sort of topology in $\mathcal{D}$, e.g. the homogeneous ball of the radius $r>0$ is defined as follows $B_{\mathbf{d}}(r)=\left\{u \in \mathcal{D}:\|u\|_{\mathbf{d}}<r\right\}$. The next two propositions are proved in Appendix.

Proposition 2: If $\boldsymbol{d}$ is monotone dilation on $\mathcal{D} \subset \boldsymbol{B}$, $z \in C((a, b), \boldsymbol{B})$ and $z(t) \in \mathcal{D} \backslash\{\boldsymbol{O}\}$ for all $t \in(a, b)$ then $\|z(\cdot)\|_{\boldsymbol{d}} \in C((a, b),(0,+\infty))$.

Proposition 3: If $\boldsymbol{d}$ is monotone and uniformly continuous on $\boldsymbol{B}$ then $\left\|u_{1}\right\|_{\boldsymbol{d}}^{\left\|G_{\boldsymbol{d}}\right\|}-\left\|u_{2}\right\|_{\boldsymbol{d}}^{\left\|G_{\boldsymbol{d}}\right\|} \leq\left\|u_{1}-u_{2}\right\|, u_{1}, u_{2} \in \boldsymbol{B} \backslash\{\boldsymbol{0}\}$. The latter inequality implies Lipschitz continuity of the homogeneous norm outside the origin if the dilation is uniformly continuous.

Definition 5: A monotone dilation $\boldsymbol{d}$ is said to be Fréchet differentiable (smooth) on $\mathcal{D} \subset \boldsymbol{B}$ if the homogeneous norm $\|\cdot\|_{\boldsymbol{d}}$ is Fréchet differentiable (smooth) on $\mathcal{D} \backslash\{\boldsymbol{0}\}$.

Let $\mathbf{d}$ be a dilation of a Hilbert space $\mathbf{H}$ and let the norm in $\mathbf{H}$ be induced by the inner product $\|\cdot\|=\sqrt{\langle\cdot, \cdot\rangle}$. If $\mathbf{d}$ is monotone on $\mathcal{D} \subset \mathcal{D}\left(G_{\mathbf{d}}\right)$ then the Fréchet derivative of the homogeneous norm $\|\cdot\|_{\mathbf{d}}$ admits the representation

$$
\left(D\|u\|_{\mathbf{d}}\right)(\cdot)=e^{-s \frac{\langle\mathbf{d}(s) \cdot, \mathbf{d}(s) u\rangle}{\left\langle G_{\mathbf{d}} \mathbf{d}(s) u, \mathbf{d}(s) u\right\rangle},}
$$

where $s \in \mathbb{R}:\|\mathbf{d}(s) u\|=1$ and $u \in \mathcal{D} \backslash\{\mathbf{0}\}$. This formula immediately follows from implicit function theorem [33, Theorem E], the identity $(D\|u\|)(\cdot)=\frac{\langle u, \cdot\rangle+\langle\cdot, u\rangle}{2\|u\|}=\frac{\langle\cdot, u\rangle}{\|u\|}$ and the property $\frac{d}{d s} \mathbf{d}(s) u=G_{\mathbf{d}} \mathbf{d}(s) u$ for $u \in \mathcal{D}\left(G_{\mathbf{d}}\right)$, which always holds for strongly continuous semigroups [32]. Therefore we have proven the following

Proposition 4: If $\left\langle u, G_{\boldsymbol{d}} u\right\rangle \neq 0$ for all $u \in \mathcal{D} \backslash\{\boldsymbol{0}\}$ then $\boldsymbol{d}$ is Fréchet smooth on $\mathcal{D} \subseteq \boldsymbol{H}$.

Remark 2 (Multi-dilations): The dilation d can combine two (or several) strongly continuous groups $\mathbf{d}(s)=$ $\mathbf{d}_{1}(s) \mathbf{d}_{2}(s)$ provided that they commute : $\mathbf{d}_{1}(s) \mathbf{d}_{2}(s)=$ $\mathbf{d}_{2}(s) \mathbf{d}_{1}(s)$ for all $s \in \mathbb{R}$. It can be shown that $G_{\mathbf{d}}=G_{\mathbf{d}_{1}}+G_{\mathbf{d}_{2}}$.

\section{Homogeneous Evolution EQuATIONS}

Let us consider nonlinear evolution equation

$$
\begin{gathered}
\dot{u}(t)=f(u(t)), \quad t>t_{0}, \\
u\left(t_{0}\right)=u_{0} \in \mathcal{D},
\end{gathered}
$$

where $t_{0} \in \mathbb{R}, u(t) \in \mathbf{B}$ and $f: \mathcal{D} \subset \mathbf{B} \rightarrow \mathbf{B}$.

Definition 6 ([14]): An operator $f: \mathcal{D} \rightarrow \boldsymbol{B}$ is said to be $\boldsymbol{d}$-homogeneous of degree $\nu$ if $\mathcal{D}$ is $\boldsymbol{d}$-homogeneous set and

$$
f(\boldsymbol{d}(s) u)=e^{\nu s} \boldsymbol{d}(s) f(u), s \in \mathbb{R}, u \in \mathcal{D},
$$

where $\boldsymbol{d}$ is a dilation in $\boldsymbol{B}$ and $\nu \in \mathbb{R}$.

The equation (5) is homogeneous if $f$ is $\mathbf{d}$-homogeneous. One has the following proposition, which is proved in Appendix D. 
Proposition 5: Let a dilation $\boldsymbol{d}$ be uniformly continuous and monotone on $\boldsymbol{B}$ and an operator $p: \boldsymbol{B} \rightarrow \boldsymbol{B}$ be $\boldsymbol{d}$ homogeneous of degree $\nu$.

- If p satisfies a Lipschitz condition on $S$ then it satisfies a Lipschitz condition on $\mathcal{K}\left(r_{1}, r_{2}\right)=\left\{u \in \boldsymbol{B}: r_{1} \leq\right.$ $\left.\|u\| \leq r_{2}\right\}$ with $0<r_{1}<r_{2}<+\infty$.

- If $p$ is Fréchet differentiable on $S$ and $\boldsymbol{d}$ is Fréchet smooth on $\boldsymbol{B}$ then $p$ is Fréchet differentiable on $\boldsymbol{B} \backslash\{\boldsymbol{0}\}$ :

$$
D p(z)=e^{\nu \ln \|z\|_{\boldsymbol{d}}} \boldsymbol{d}\left(\ln \|z\|_{\boldsymbol{d}}\right) D p\left(z_{0}\right) \boldsymbol{d}\left(-\ln \|z\|_{\boldsymbol{d}}\right),
$$

where $D p(z) \in \mathcal{L}(\boldsymbol{B})$ and $D p\left(z_{0}\right) \in \mathcal{L}(\boldsymbol{B})$ are Fréchet derivatives of $p$ at $z \in \boldsymbol{B} \backslash\{\boldsymbol{0}\}$ and $z_{0} \in S$, respectively.

The latter properties of the homogeneous operator allows us to refine the classical results about existence of solutions of quasi-linear evolution equations.

Corollary 1 (On Homogeneous Perturbation): Let

- $A: \mathcal{D}(A) \subset \boldsymbol{B} \rightarrow \boldsymbol{B}$ be a generator of a strongly continuous semigroup $\left\{e^{A t}\right\}_{t \in \mathbb{R}_{+}}$in $\boldsymbol{B}$ and $M_{1}\left(1-\sigma_{1}(t)\right)\|u\| \leq$ $\left\|e^{A t} u\right\| \leq M_{2}\left(1+\sigma_{2}(t)\right)\|u\|$ for $u \in \boldsymbol{B}$ and $t \in[0, T]$, where $M_{i}>0$, $\sigma_{i}: \mathbb{R}_{+} \rightarrow \mathbb{R}_{+}$are continuous nondecreasing functions, $\sigma_{i}(0)=0, i=1,2$ and $T>0$;

- $\boldsymbol{d}$ be a uniformly continuous monotone dilation on $\boldsymbol{B}$;

- an operator $p: \boldsymbol{B} \rightarrow \boldsymbol{B}$ be $\boldsymbol{d}$-homogeneous of degree $\nu$ and satisfy Lipschitz condition on $S$,

then the nonlinear evolution equation (5) with $f=A+p$ has a unique mild solution

$$
u(t)=e^{A t} u(0)+\int_{0}^{t} e^{A(t-s)} p(u(s)) d s
$$

for any $u(0) \in \boldsymbol{B} \backslash\{\boldsymbol{0}\}$ with $t \in\left(0, t^{\max }\right)$, where $t^{\max }=+\infty$ or $\|u(t)\| \rightarrow+\infty$ or $\|u(t)\| \rightarrow 0$ as $t \rightarrow t^{\max }$.

The proof of this corollary follows from [32, Theorem 1.4, Chapter 6] and Proposition 5.

Corollary 2 (Regularity): If all conditions of Corollary 1 hold, the dilation $\boldsymbol{d}(s)$ is Fréchet smooth on $\boldsymbol{B}$ and $p: \boldsymbol{B} \rightarrow \boldsymbol{B}$ is Fréchet smooth on $S$ then the solution (8) is classical ${ }^{2}$ one. The proof of this corollary can be derived using [32, Theorem 1.5, Chapter 6] and Proposition 5.

Example: Let $\mathbf{B}=L^{2}(\mathbb{R}, \mathbb{R}), A=\frac{\partial^{2}}{\partial x^{2}}, p(u)=k\|u\|^{\alpha-1} u$ and $k \in \mathbb{R}, \alpha \in \mathbb{R}_{+}$. Using the heat kernel we can show that $\left\{e^{A t}\right\}$ satisfies conditions of Corollary 1. The operator $p$ is d-homogeneous for uniformly continuous Fréchet smooth dilation $\mathbf{d}(s)=e^{s} I, G_{\mathbf{d}}=I \in \mathcal{L}(\mathbf{B})$, In addition, $p$ is Fréchet smooth on $S$. Therefore, all conditions of Corollary 2 hold, so the quasi-linear heat equation [34]

$$
\begin{gathered}
\frac{\partial u}{\partial t}=\frac{\partial^{2} u}{\partial x^{2}}+k u\|u\|^{\alpha-1}, \quad t \in \mathbb{R}_{+}, \quad x \in \mathbb{R}, \\
u: \mathbb{R}_{+} \times \mathbb{R} \rightarrow \mathbb{R} \text { and } k, \alpha \in \mathbb{R} .
\end{gathered}
$$

has a unique classical solution defined at least locally on $\left[0, t_{\max }\right)$ for any non-trivial initial condition $u(0) \in \mathcal{D}(A) \backslash \mathbf{0}$.

\section{Homogeneous Finite-Time Control}

Let us consider the following control system

$$
\dot{u}(t)=A u(t)+B \xi(u(t)), \quad t>0
$$

\footnotetext{
${ }^{2}$ According to [32] a continuous function $u:[0, T] \rightarrow \mathbf{B}$ is called classical solution to (5) if $u(t) \in \mathcal{D}(A)$ for $0<t \leq T, u$ is continuously differentiable on $0<t<T$ and satisfies (5), (6).
}

$$
u(0)=u_{0} \in \mathcal{D}(A),
$$

where the operators $A: \mathcal{D}(A) \rightarrow \mathbf{H}$ and $B: \mathcal{D}(B) \subset \mathbf{B} \rightarrow$ $\mathbf{H}$ are linear, $\mathbf{B}$ is a Banach space, $\mathbf{H}$ is a Hilbert space with the inner product $\langle\cdot, \cdot\rangle, \mathcal{D}(A)$ and $\mathcal{D}(B)$ are domains of the operators, $u(t) \in \mathcal{D}(A) \subset \mathbf{H}$ is the system state, $\xi: \mathcal{D}(A) \rightarrow$ $\mathcal{D}(B)$ is a bounded (locally or globally) feedback control to be designed in order to steer any solution of the closed-loop system to zero in a finite time. The problem of bounded finitetime stabilizing control design for the considered system has been studied, for example, in [35]. This problem also appears in the context of sliding mode control of PDEs [36], [37].

In [38], [17] finite-time stabilizing feedback design is proposed for ODE using a combination of the implicit Lyapunov function method with the homogeneity approach. The design procedure is composed of two steps. First, a linear stabilizing feedback is designed together with the corresponding quadratic Lyapunov function. Next, homogeneous dilation is applied in order to construct the finite-time stabilizing feedback and the corresponding implicit Lyapunov function. In this paper, we follow the same scheme and use the homogeneous norm $\|u\|_{\mathbf{d}}$ defined by (2) as a Lyapunov function. The specific feature of the infinite dimensional case is multi-dilation application for finite-time control design.

Theorem 1 (On homogeneous finite-time feedback): If

A) the operator $A: \mathcal{D}(A) \subset \boldsymbol{H} \rightarrow \boldsymbol{H}$ is an infinitesimal generator of a strongly continuous semigroup on $\boldsymbol{H}$ and $B: \mathcal{D}(B) \subset \boldsymbol{B} \rightarrow \boldsymbol{H}$ is a linear bounded operator,

B) the dilation $\boldsymbol{d}=\boldsymbol{d}_{1} \boldsymbol{d}_{2}$ is monotone on $\boldsymbol{H}$ and combined of two groups $\boldsymbol{d}_{1}(s) \boldsymbol{d}_{2}(s)=\boldsymbol{d}_{2}(s) \boldsymbol{d}_{1}(s), s \in \mathbb{R}$ such that

- $\boldsymbol{d}_{1}$ is a monotone uniformly continuous dilation,

- $\boldsymbol{d}_{2}$ is a strongly continuous group on $\boldsymbol{H}$ :

$$
\left\|\boldsymbol{d}_{2}(s)\right\|_{\mathcal{L}}^{\boldsymbol{H}} \leq M\left\{\begin{array}{ll}
e^{\omega^{+} s} & \text { if } s \geq 0, \\
e^{\omega^{-} s} & \text { if } s<0,
\end{array} M>0, \omega^{+}, \omega^{-} \in \mathbb{R},\right.
$$

- the operator $A: \mathcal{D}(A) \subset \boldsymbol{B} \rightarrow \boldsymbol{B}$ is $\boldsymbol{d}$ homogeneous of a negative degree $-\mu$ and $\mathcal{D}(A) \subset$ $\mathcal{D}\left(G_{\boldsymbol{d}}\right)$, where $\mu>0$ and $G_{\boldsymbol{d}}$ is the generator of $\boldsymbol{d}$,

C) there exists a linear bounded operator $K: \boldsymbol{H} \rightarrow \boldsymbol{B}$ such that $B K(\mathcal{D}(A)) \subset \mathcal{D}(A)$ and

$$
\begin{gathered}
\left\langle\left(A+B K+\alpha G_{\boldsymbol{d}}\right) z, z\right\rangle+\left\langle z,\left(A+B K+\alpha G_{\boldsymbol{d}}\right) z\right\rangle \leq 0, \\
2 \beta\|z\|^{2} \leq\left\langle G_{\boldsymbol{d}} z, z\right\rangle+\left\langle z, G_{\boldsymbol{d}} z\right\rangle,
\end{gathered}
$$

for all $z \in \mathcal{D}(A)$, where $\alpha, \beta>0$ are some constants,

D) there exists a nonnegative number $\nu \geq 0$ such that the operator $B K$ satisfies $e^{-\nu s} \boldsymbol{d}(s) B K z=B K \boldsymbol{d}_{2}(s) z$ for all $z \in \mathcal{D}(A)$,

then the feedback $\xi: \boldsymbol{H} \rightarrow \boldsymbol{B}$ defined as

$$
\xi(u)=\left\{\begin{array}{cl}
\|u\|_{\boldsymbol{d}}^{\nu-\mu} K \boldsymbol{d}_{1}\left(-\ln \left(\|u\|_{\boldsymbol{d}}\right)\right) u & \text { if } u \neq 0, \\
0 & \text { if } u=0
\end{array}\right.
$$

is locally bounded on $\boldsymbol{H} \backslash\{\boldsymbol{0}\}$, Fréchet differentiable at any point $u \in \mathcal{D}(A) \backslash\{\boldsymbol{0}\}$, continuous at $\boldsymbol{O} \in \boldsymbol{H}$ if $\nu+\omega^{-}>\mu$, globally bounded $\left(\|\xi(\cdot)\| \leq M\|K\|_{\mathcal{L}}\right)$ if $\nu-\mu=\omega^{+} \geq$ $-\omega-, B \xi(\mathcal{D}(A)) \subset \mathcal{D}(A)$ and for any $u(0)=u_{0} \in$ $\mathcal{D}(A) \backslash\{\boldsymbol{0}\}$ the closed-loop system (9), (11) has unique classical solution $u(t) \in \mathcal{D}(A)$, such that $\|u(t)\| \rightarrow 0$ as $t \rightarrow T\left(u_{0}\right) \leq \frac{\left\|u_{0}\right\|_{d}^{\mu}}{\alpha \mu}$. 
If the first inequality in C) becomes an identity then $T\left(u_{0}\right)=\frac{\left\|u_{0}\right\|_{\mathbf{d}}^{\mu}}{\alpha \mu}$. The proof of Theorem 1 is given in [39], where non-homogeneous operators $A$ are treated. For $\mathbf{H}=\mathbb{R}^{n}$ an analogue of Theorem 1 has been proven in [38].

Remark 3: Theorem 1 asks the operator $A$ to be homogeneous of negative degree, it is well known that linear operators are always homogeneous with zero degree. Indeed, for any linear operator $A$ we have $A \boldsymbol{d}(s)=\boldsymbol{d}(s) A$ if $\boldsymbol{d}(s)=$ $e^{s} I$. However, there exist linear operators, which may have also positive and negative homogeneity degrees. For example, if $B=\mathbb{R}^{n}$ then the operator $A=\left(\begin{array}{cccccc}0 & 1 & 0 & \ldots & 0 & 0 \\ 0 & 0 & 1 & \ldots & 0 & 0 \\ \ldots & \ldots & \ldots & \ldots & \ldots & 0 \\ 0 & 0 & 0 & \ldots & 0 & 1 \\ 0 & 0 & 0 & \ldots & 0 & 0\end{array}\right)$ is $\boldsymbol{d}$-homogeneous with positive degree $\nu=1$ if $\ddot{\boldsymbol{d}}(s)=$ $\operatorname{diag}\left\{e^{i s}\right\}_{i=1}^{n}$ and $\boldsymbol{d}$-homogeneous with negative degree $\nu=$ -1 if $\boldsymbol{d}(s)=\operatorname{diag}\left\{e^{(n-i) s}\right\}_{i=1}^{n}$. For $\boldsymbol{B}=L^{2}(\mathbb{R}, \mathbb{R})$ the Laplace operator $A=\frac{\partial^{2}}{\partial x^{2}}$ has similar property: it is $\boldsymbol{d}$ homogeneous of degree $2 \mu$ if $(\boldsymbol{d}(s) u)(x)=e^{s} u\left(e^{\mu s} x\right)$, where $\mu \in \mathbb{R}$ is an arbitrary number. Theorem 1 can be applied to plant models with such operators.

\section{Examples of Finite-time Control Design}

\section{A. Example 1: Heat Equation on $\mathbb{R}_{+}$}

Let us denote $\mathbf{H}=H^{0}\left(\mathbb{R}_{+}, \mathbb{R}\right), B=I \in \mathcal{L}(\mathbf{H})$ and consider the operator $A=\frac{\partial^{2}}{\partial x^{2}}: \mathcal{D}(A) \subset \mathbf{H} \rightarrow \mathbf{H}$ with the domain $D(A)=\left\{z \in H^{2}\left(\mathbb{R}_{+}, \mathbb{R}\right): z(0)=0\right\}$ and $K=-\rho I$.

Let us consider the dilation $\mathbf{d}(s)=\mathbf{d}_{1}(s) \mathbf{d}_{2}(s)$, where $\mathbf{d}_{1}(s)=e^{\nu s} I$ with $\nu>0$ is uniformly continuous monotone dilation on $\mathbf{H}, G_{\mathbf{d}_{1}} u=\nu u$ and $\left(\mathbf{d}_{2}(s) u\right)(x)=u\left(e^{-\mu s} x\right), x \in$ $\mathbb{R}_{+}, \mu>0$ is strongly continuous group on $\mathbf{H},\left(G_{\mathbf{d}_{2}} u\right)(x)=$ $-\mu x u^{\prime}(x), x \in \mathbb{R}_{+}$. The operator $A$ is d-homogeneous of degree $-2 \mu$ and $B K$ satisfies condition D) of Theorem 1.

Taking into account that $G_{\mathbf{d}} u=\nu u-\mu x u^{\prime}$ we derive $\left(A+B K+\alpha G_{\mathbf{d}}\right) z=z^{\prime \prime}-\rho z+\alpha \nu z-\alpha \mu x z^{\prime}$. Since for any $z \in \mathcal{D}(A)$ we have $\left\langle x z^{\prime}, z\right\rangle=\left.0.5 x z^{2}(x)\right|_{0} ^{+\infty}-0.5\langle z, z\rangle=$ $-0.5\langle z, z\rangle$ then for $\rho \geq \alpha(\nu+0.5 \mu)$ we derive $\langle(A+$ $\left.\left.B K+\alpha G_{\mathbf{d}}\right) z, z\right\rangle=-\left\langle z^{\prime}, z^{\prime}\right\rangle-(\rho-\alpha(\nu+0.5 \mu))\langle z, z\rangle \leq$ 0 if $z \in \mathcal{D}(\tilde{A})$. Therefore, all conditions of Theorem 1 hold and the distributed feedback control (11) stabilizes at zero any solution of (9) in a finite-time. Let us find an explicit representation of the control law. Since $\|\mathbf{d}(s) u\|=$ $e^{(\nu+0.5 \mu) s} \sqrt{\int_{\mathbb{R}_{+}} u^{2}(x) d x}$ then $\|u\|_{\mathbf{d}}=\|u\|^{1 /(\nu+0.5 \mu)}$. So, according to Theorem 1 the finite-time stabilizing feedback

$$
\xi(u)=-\rho\|u\|^{\frac{-\mu}{\nu+0.5 \mu}} u
$$

is continuously Fréchet differentiable on $\mathcal{D}(A) \backslash\{\mathbf{0}\}$, continuous at $\mathbf{0}$ if $\nu>0.5 \mu$ and globally bounded (but discontinuous at $\mathbf{0})$ if $\nu=0.5 \mu$.

\section{B. Example 2 : Wave Equation on $\mathbb{R}$}

The distributed finite-time control design procedure is based on Theorem 1. We rewrite the wave equation in the form (9) and check all conditions of the theorem.

A) Let us denote $\mathbf{H}=H^{1}(\mathbb{R}, \mathbb{R}) \times H^{0}(\mathbb{R}, \mathbb{R})$ and $u=\left(u_{1}, u_{2}\right)^{\top} \in \mathbf{H}, \quad A=\left(\begin{array}{cc}O & I \\ \frac{\partial^{2}}{\partial x^{2}} & O\end{array}\right): \mathcal{D}(A) \subset \mathbf{H} \rightarrow \mathbf{H}$, where $\mathcal{D}(A)=H^{2}(\mathbb{R}, \mathbb{R}) \times H^{1}(\mathbb{R}, \mathbb{R}), O \in \mathcal{L}\left(H^{0}(\mathbb{R}, \mathbb{R})\right)$ and $I \in \mathcal{L}\left(H^{0}(\mathbb{R}, \mathbb{R})\right)$ are zero and identity operators, respectively, $B=\left(\begin{array}{c}O \\ I\end{array}\right): H^{0}(\mathbb{R}, \mathbb{R}) \rightarrow \mathbf{H}$. It is known [32] that $A$ is a generator of strongly continuous semigroup in $\mathbf{H}$.

Let us define the norm $\|\cdot\|=\sqrt{\langle\cdot, \cdot\rangle}$ in $\mathbf{H}$ by means of the following inner product

$$
\langle u, v\rangle=\int_{\mathbb{R}} u^{\top}(x) P v(x)+P_{22} u_{1}^{\prime}(x) v_{1}^{\prime}(x) d x,
$$

with $P=\left(\begin{array}{ll}P_{11} & P_{12} \\ P_{12} & P_{22}\end{array}\right)=X^{-1}$, where $X=\left(\begin{array}{ll}X_{11} & X_{12} \\ X_{12} & X_{22}\end{array}\right) \in \mathbb{R}^{2 \times 2}$ is a positive definite solution of the system of linear matrix inequalities (LMIs):

$$
\begin{gathered}
\left(\begin{array}{cc}
\nu+\mu & 1 \\
0 & \nu
\end{array}\right) X+X\left(\begin{array}{cc}
\nu+\mu & 0 \\
1 & \nu
\end{array}\right)+y^{\top} b^{\top}+b y+\mu X=0 \\
\left(\begin{array}{rr}
\nu+\mu & 0 \\
0 & \nu
\end{array}\right) X+X\left(\begin{array}{rr}
\nu+\mu & 0 \\
0 & \nu
\end{array}\right)>0, \quad X>0
\end{gathered}
$$

where $\nu, \mu \in \mathbb{R}_{+}, b=(0,1)^{\top}, y^{\top}=\left(y_{1}, y_{2}\right)^{\top} \in \mathbb{R}^{2}$. The system of LMIs is always feasible [17] with respect to $X$ and $y$. Since $(\nu+1.5 \mu) X_{11}+X_{12}=0$ then it can be shown that $0<(\nu+1.5 \mu) P_{22}=P_{12}$. Let the operator $K$ be defined as $K z=y P^{-1} z$ for any $z \in \mathbf{H}$.

B) Let us introduce the dilation $\mathbf{d}=\mathbf{d}_{1} \mathbf{d}_{2}$ on $\mathbf{H}$, where $\mathbf{d}_{1}(s) u=\left(\begin{array}{cc}e^{(\nu+\mu) s} & 0 \\ 0 & e^{\nu s}\end{array}\right) u, \quad\left(\mathbf{d}_{2}(s) u\right)(x)=u\left(e^{-\mu s} x\right), \quad u \in \mathbf{H}$. The dilation $\mathbf{d}$ has the generator $G_{\mathbf{d}}=G_{\mathbf{d}_{1}}+G_{\mathbf{d}_{2}}$, where $G_{\mathbf{d}_{1}} u=\left(\begin{array}{cc}\nu+\mu & 0 \\ 0 & \nu\end{array}\right) u, G_{\mathbf{d}_{2}} u=-\mu x u^{\prime}$ for $u \in \mathcal{D}(A)$. The dilations $\mathbf{d}$ and $\mathbf{d}_{1}$ are monotone on $\mathbf{H}$. Indeed, since

$\left\|\mathbf{d}_{1}(s) u\right\|^{2}=\int_{\mathbb{R}} u^{\top}(x) \mathbf{d}_{1}(s) P \mathbf{d}_{1}(s) u(x)+P_{22} e^{2(\nu+\mu) s}\left(u_{1}^{\prime}(x)\right)^{2} d x$

then due to LMIs one holds $\frac{d}{d s}\left\|\mathbf{d}_{1}(s) u\right\|^{2}=$ $\int_{\mathbb{R}} u(x)^{\top} \mathbf{d}_{1}(s)\left(G_{\mathbf{d}_{1}} P^{-1}+P^{-1} G_{\mathbf{d}_{1}}\right) \mathbf{d}_{1}(s) u(x)+2(\nu+$ $\mu) e^{2(\nu+\mu) s}\left(u_{1}^{\prime}(x)\right)^{2} d x>0$ for any $s \in \mathbb{R}, u \in \mathcal{D}(A)$. The monotonicity of the dilation $\mathbf{d}$ can be proven analogously taking into account

$$
\left\|\mathbf{d}_{2}(s) u\right\|^{2}=\int_{\mathbb{R}} e^{\mu s} u^{\top}(x) P u(x)+P_{22} e^{-\mu s}\left(u_{1}^{\prime}(x)\right)^{2} d x .
$$

The dilation $\mathbf{d}_{1}$ is uniformly continuous on $\mathbf{H}$ and the dilation $\mathbf{d}$ is Fréchet smooth on $\mathcal{D}(A)$. The operator $A$ is d-homogeneous of degree $-\mu<0$. Indeed, $(A \mathbf{d}(s) u)(x)=$ $A\left(\begin{array}{c}e^{(\nu+\mu) s} u_{1}\left(e^{-\mu s} x\right) \\ e^{\nu s} u_{2}\left(e^{-\mu s} x\right)\end{array}\right)=\left(\begin{array}{c}e^{\nu s} u_{2}\left(e^{-\mu s} x\right) \\ e^{(\nu-\mu) s} u_{1}^{\prime \prime}\left(e^{-\mu s} x\right)\end{array}\right)=e^{-\mu s}(\mathbf{d}(s) A u)(x)$.

C) Given $z \in \mathcal{D}(A)$ and $A_{0}=\left(\begin{array}{ll}0 & I \\ 0 & 0\end{array}\right)$ we have $\langle z,(A+B K+$ $\left.\left.G_{\mathbf{d}}\right) z\right\rangle=\left\langle z,\left(A_{0}+B K+G_{\mathbf{d}_{1}}\right) z\right\rangle+\left\langle z,\left(\begin{array}{c}\mathbf{0} \\ z_{1}^{\prime \prime}\end{array}\right)\right\rangle+\left\langle z, G_{\mathbf{d}_{2}} z\right\rangle \leq 0$, since $\left\langle z,\left(\begin{array}{c}\mathbf{0} \\ z_{1}^{\prime \prime}\end{array}\right)\right\rangle=\int_{\mathbb{R}} P_{12}\left(z_{1}^{\prime}(x)\right)^{2}-P_{22} z_{1}^{\prime}(x) z_{2}^{\prime}(x) d x$, $\left\langle z, G_{\mathbf{d}_{2}} z\right\rangle=-\mu\left\langle z, x z^{\prime}\right\rangle=\frac{\mu}{2}\langle z, z\rangle$ and due to LMI one has $\left\langle z,\left(A_{0}+B K+G_{\mathbf{d}_{1}}\right) z\right\rangle=-\frac{\mu}{2}\langle z, z\rangle+\left[P_{22} \int_{\mathbb{R}} z_{1}^{\prime}(x)((\nu+\right.$ н) $\left.z_{1}^{\prime}(x)+z_{2}^{\prime}(x)\right) d x$, where $0<(\nu+1.5 \mu) P_{22}=P_{12}$.

D) Since $e^{-\nu s} \mathbf{d}(s) B K=B K \mathbf{d}_{2}(s)$ then by Theorem 1 wave equation admits homogeneous finite-time control (11), so closed-loop system is homogeneous of degree $-\mu$.

In general the homogeneous norm $\|\cdot\|_{\mathbf{d}}$ (as well as feedback (11)) is defined implicitly, see (2). Using the ideas of [40] for $\nu=\frac{\mu}{2}$ the homogeneous norm can be found explicitly as a unique real non-negative solution to the quartic equation $V^{4}=a V^{2}+b V+c$ that is equivalent to $\|\mathbf{d}(-s) u\|=1$ if $V=e^{\mu s}, a=p_{22} \int_{\mathbb{R}} u_{2}^{2}(x)+\left(u_{1}^{\prime}(x)\right)^{2} d x$, 
$b=2 p_{12} \int_{\mathbb{R}} u_{1}(x) u_{2}(x) d x$ and $c=p_{11} \int_{\mathbb{R}} u_{1}^{2}(x) d x$. In particular, for $\mu=1$ using Ferrari formulas we derive

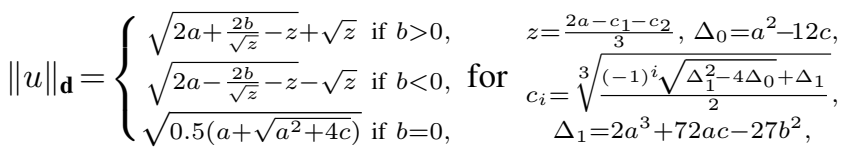

so the feedback (11) for wave equation can be found in the explicit form: $\xi(u)=K \operatorname{diag}\left(\|u\|_{\mathbf{d}}^{-2},\|u\|_{\mathbf{d}}^{-1}\right) u$.

\section{Conclusions}

The paper introduces a novel framework for control design based on generalized homogeneity of operators in Banach and Hilbert spaces. The so-called monotone dilations and homogeneous norms are studied. The conventional solution existence theorems for quasi-linear equations are refined for the case of homogeneous perturbations.

The universal homogeneous distributed finite-time control is presented for evolution equations with plant model given by a homogeneous linear (possibly unbounded) operator in a Hilbert space. The presented results can be extended to some Banach spaces. Currently, the proof of Theorem 1 is essentially based on Fréchet smoothness of the homogeneous norm, which is also required for existence of classical solutions of the closed-loop system. This property can possibly be guaranteed for super-reflexive Banach spaces. The extension to other Banach spaces will need another solution existence/uniqueness theorem to be proven.

As examples of the main theorem the distributed finitetime control has been designed for heat and wave equations on unbounded domains. The case of bounded domains is treated in [39], where Theorem 1 is refined for homogeneous extensions of operators. The developed homogeneous framework looks promising for design of finite-time control for linear and non-linear PDEs.

\section{APPENDIX}

\section{A. Proof of Proposition 1}

1) If $u \in S_{\mathbf{d}}(1)$ then $1=\|u\|=\|\mathbf{d}(-s) \mathbf{d}(s) u\| \leq$ $\|\mathbf{d}(-s)\|_{\mathcal{L}}^{\mathcal{D}} \cdot\|\mathbf{d}(s) u\|$ for any $s>0$. Hence, $\|\mathbf{d}(s) u\| \geq$ $\frac{1}{\|\mathbf{d}(-s)\|_{\mathcal{L}}^{\mathcal{D}}}$ for any fixed $s>0$ and arbitrary $u \in S_{\mathbf{d}}(1)$. Due to strong contraction (monotonicity) condition, $1<\frac{1}{\|\mathbf{d}(-s)\|_{\mathcal{L}}^{\mathcal{D}}} \leq$ $\inf _{u \in S_{\mathbf{d}}(1)}\|\mathbf{d}(s) u\|=\lfloor\mathbf{d}(s)\rfloor_{\mathcal{L}}^{\mathcal{D}}$ for any $s>0$.

2) Existence of the pair $\left(s_{0}, u_{0}\right)$ is provided in [14]. Let us prove the uniqueness of this pair in the case of monotone dilation. Suppose the contrary, i.e. for some $u \in \mathcal{D}$ there exist $\left(s_{1}, u_{1}\right) \in \mathbb{R} \times S_{\mathbf{d}}(1)$ and $\left(s_{2}, u_{2}\right) \in \mathbb{R} \times S_{\mathbf{d}}(1)$ such that $\left(s_{1}, u_{1}\right) \neq\left(s_{2}, u_{2}\right)$ and $\mathbf{d}\left(s_{1}\right) u_{1}=u=\mathbf{d}\left(s_{2}\right) u_{2}$. If $s_{1}=s_{2}$ then $d\left(s_{1}\right) u_{1}=d\left(s_{2}\right) u_{2} \Rightarrow u_{1}=\mathbf{d}\left(-s_{1}+s_{2}\right) u_{2}=$ $u_{2}$ and we obtain the contradiction. If $s_{1} \neq s_{2}$ then $u_{1}=$ $d\left(s_{2}-s_{1}\right) u_{2}$. Without loss of generality we may assume $s_{2}-s_{1}<0$ (otherwise we consider the equivalent identity $\left.d\left(s_{1}-s_{2}\right) u_{1}=u_{2}\right)$. In this case $1=\left\|u_{1}\right\| \leq\left\|\mathbf{d}\left(s_{2}-s_{1}\right)\right\|_{\mathcal{L}}^{\mathcal{D}}$. $\left\|u_{2}\right\|=\left\|\mathbf{d}\left(s_{2}-s_{1}\right)\right\|_{\mathcal{L}}^{\mathcal{D}}$. The obtained inequality contradicts the monotonicity condition (1).

3) Strong continuity of the semigroup $\mathbf{d}$ implies continuity of $\|\mathbf{d}(\cdot) u\|$ for any $u \in \mathcal{D}$. For any $u \in \mathcal{D} \backslash\{\boldsymbol{0}\}$ and any $s_{1}<$ $s_{2}$ one have $\left\|\mathbf{d}\left(s_{1}\right) u\right\|-\left\|\mathbf{d}\left(s_{2}\right) u\right\|=\left\|\mathbf{d}\left(s_{1}\right) u\right\|-\| \mathbf{d}\left(s_{2}-\right.$ $\left.s_{1}\right) \mathbf{d}\left(s_{1}\right) u\left\|\leq\left(1-\left\lfloor\mathbf{d}\left(s_{2}-s_{1}\right)\right\rfloor_{\mathcal{L}}^{\mathcal{D}}\right)\right\| \mathbf{d}\left(s_{1}\right) u \|$, The property 1) immediately yields the strict monotonicity.

4) Let us notice that monotonicity of $\|\mathbf{d}(\cdot)\|_{\mathcal{L}}^{\mathcal{D}}$ and $\lfloor\mathbf{d}(\cdot)\rfloor_{\mathcal{L}}^{\mathcal{D}}$ follow from 3 ). Let us show that the considered functions are lower and upper semicontinuous at $s=0$, respectively. Taking into account their monotonicity we have

$\|\mathbf{d}(s)\|_{\mathcal{L}}^{\mathcal{D}} \rightarrow 1+c^{+}$and $\left.\lfloor\mathbf{d}(\cdot)\rfloor\right\rfloor_{\mathcal{D}}^{\mathcal{D}} \rightarrow 1+c_{+}$as $s \rightarrow 0^{+}$,
$\|\mathbf{d}(s)\|_{\mathcal{L}}^{\mathcal{D}} \rightarrow 1-c^{-}$and $\lfloor\mathbf{d}(\cdot)\rfloor{ }_{\mathcal{L}}^{\mathcal{L}} \rightarrow 1-c_{-}$as $s \rightarrow 0^{-}$, where $c^{+}, c^{-}, c_{+}, c_{-} \in \mathbb{R}_{+}$. For $u \in S_{\mathbf{d}}(1)$ and $s \in \mathbb{R}$ we have $1=\|u\|=\|\mathbf{d}(s) \mathbf{d}(-s) u\| \leq\|\mathbf{d}(s)\|_{\mathcal{L}}^{\mathcal{D}} \cdot\|\mathbf{d}(-s) u\|$ and $1=\|u\|=\|\mathbf{d}(s) \mathbf{d}(-s) u\| \geq\lfloor\mathbf{d}(s)\rfloor_{\mathcal{L}}^{\mathcal{D}} \cdot\|\mathbf{d}(-s) u\|$.

Taking inf and sup on both sides of the obtained inequalities we derive $1 \leq\|\mathbf{d}(s)\|_{\mathcal{L}} \cdot\lfloor\mathbf{d}(-s)\rfloor_{\mathcal{L}}^{\mathcal{D}}$ and $1 \geq$ $\lfloor\mathbf{d}(s)\rfloor_{\mathcal{L}}^{\mathcal{D}} \cdot\|\mathbf{d}(-s)\|_{\mathcal{L}}^{\mathcal{D}}$ for any $s \in \mathbb{R}$. For $s \rightarrow 0^{+}$we derive $\left(1+c_{+}\right)\left(1-c^{-}\right) \leq 1 \leq\left(1+c^{+}\right)\left(1-c_{-}\right)$, but for $s \rightarrow 0^{-}$ we obtain $\left(1-c_{-}\right)\left(1+c^{+}\right) \leq 1 \leq\left(1-c^{-}\right)\left(1+c_{+}\right)$. This immediately implies $1=\left(1-c_{-}\right)\left(1+c^{+}\right)=\left(1-c^{-}\right)\left(1+c_{+}\right)$. Since $\|\mathbf{d}(s)\|_{\mathcal{L}}^{\mathcal{D}}=\|\mathbf{d}(2 s) \mathbf{d}(-s)\|_{\mathcal{L}}^{\mathcal{D}} \leq\|\mathbf{d}(2 s)\|_{\mathcal{L}}^{\mathcal{D}} \cdot\|\mathbf{d}(-s)\|_{\mathcal{L}}^{\mathcal{D}}$ then taking into account monotonicity proven above we derive $\|\mathbf{d}(s)\|_{\mathcal{L}}^{\mathcal{D}} \leq\left(1-c^{-}\right)\|\mathbf{d}(2 s)\|_{\mathcal{L}}^{\mathcal{D}}$. For $s \rightarrow 0^{+}$we obtain $1+c^{+} \leq\left(1-c^{-}\right)\left(1+c^{+}\right)$, i.e. $c^{-}=0$ as well as $c_{+}=0$. Finally, using the group property it can be easily shown that $\|\mathbf{d}(\cdot)\|_{\mathcal{L}}^{\mathcal{D}}: \mathbb{R} \rightarrow \mathbb{R}_{+}$is lower semicontinuous, but $\lfloor\mathbf{d}(\cdot)\rfloor_{\mathcal{L}}^{\mathcal{D}}: \mathbb{R} \rightarrow \mathbb{R}_{+}$is upper semicontinuous on $\mathbb{R}$.

\section{B. Proof of Proposition 2}

If $z \in C((a, b), \mathbf{B})$ and $z(t) \in \mathcal{D} \backslash\{0\}$ then the function $q_{z}: \mathbb{R} \times(a, b) \rightarrow \mathbb{R}, q_{z}(s, t)=\|\mathbf{d}(-s) z(t)\|-1$ is continuous on both variables and strictly monotone on the first variable for any $t \in(a, b)$ (see, Proposition 1). Hence, implicit function theorem [41, Theorem 1.1] implies that there exists $\tilde{s}_{z}:(a, b) \rightarrow \mathbb{R}$ such that $\tilde{s}_{z} \in C((a, b), \mathbb{R})$ and $q_{z}(\tilde{s}(t), t)=0$ for all $t \in(a, b)$. Hence, $\|z(\cdot)\|_{\mathbf{d}}=e^{\tilde{s}_{z}(\cdot)} \in$ $C((a, b),(0,+\infty))$.

\section{Proof of Proposition 3}

By definition of homogeneous norm we have $\left\|u_{i}\right\|_{\mathbf{d}}=e^{s_{i}}$, where $\left\|\mathbf{d}\left(-s_{i}\right) u_{i}\right\|=1, s_{i} \in \mathbb{R}, i=1,2$. Since $\left\|\mathbf{d}\left(-s_{1}\right) u_{1}\right\|=$ $\left\|\mathbf{d}\left(-s_{1}\right)\left(u_{1}-u_{2}\right)+\mathbf{d}\left(-s_{1}+s_{2}\right) \mathbf{d}\left(-s_{2}\right) u_{2}\right\| \leq e^{-\left\|G_{\mathbf{d}}\right\| s_{1}} \| u_{1}-$ $u_{2} \|+e^{-\left\|G_{\mathbf{d}}\right\|\left(s_{1}-s_{2}\right)}$ then $e^{\left\|G_{d}\right\| s_{1}}-e^{\left\|G_{d}\right\| s_{2}} \leq\left\|u_{1}-u_{2}\right\|$. On the other hand, $1=\left\|\mathbf{d}\left(-s_{2}\right) u_{2}\right\|=\| \mathbf{d}\left(-s_{2}\right)\left(u_{2}-\right.$ $\left.u_{1}\right)+\mathbf{d}\left(-s_{2}+s_{1}\right) \mathbf{d}\left(-s_{1}\right) u_{1}\left\|\leq e^{-\left\|G_{\mathbf{d}}\right\| s_{2}}\right\| u_{1}-u_{2} \|+$ $e^{-\left\|G_{\mathbf{d}}\right\|\left(s_{2}-s_{1}\right)}$ and $e^{\left\|G_{\mathbf{d}}\right\| s_{2}}-e^{\left\|\bar{G}_{\mathbf{d}}\right\| s_{1}} \leq\left\|u_{1}-u_{2}\right\|$. So, $\left|\left\|u_{1}\right\|_{\mathbf{d}}^{\left\|G_{\mathbf{d}}\right\|}-\left\|u_{2}\right\|_{\mathbf{d}}^{\left\|G_{\mathbf{d}}\right\|}\right|=\left|e^{\left\|G_{d}\right\| s_{1}}-e^{\left\|G_{d}\right\| s_{2}}\right| \leq\left\|u_{1}-u_{2}\right\|$.

\section{Proof of Proposition 5}

I. Due to the relation (3) between norms there exists $s_{\min }, s_{\max } \in \mathbb{R}$ such that $\|u\|_{\mathbf{d}} \in\left[e^{s_{\text {min }}}, e^{s_{\text {min }}}\right]$ for all $u \in \mathcal{K}\left(r_{1}, r_{2}\right)$. If $z_{1}, z_{2} \in \mathcal{K}\left(r_{1}, r_{2}\right)$ then there exist $z_{1}^{0}, z_{2}^{0} \in S$ and $s_{1}, s_{2} \in \mathbb{R}$ such that $z_{1}=\mathbf{d}\left(s_{1}\right) z_{1}^{0}$ and $z_{2}=\mathbf{d}\left(s_{2}\right) z_{2}^{0}$. Hence, due to homogeneity of $p$ we derive $\left\|p\left(z_{1}\right)-p\left(z_{2}\right)\right\|=\left\|e^{\nu s_{1}} \mathbf{d}\left(s_{1}\right) p\left(z_{1}^{0}\right)-e^{\nu s_{2}} \mathbf{d}\left(s_{2}\right) p\left(z_{2}^{0}\right)\right\|=$ $\| e^{\nu s_{1}} \mathbf{d}\left(s_{1}\right) p\left(z_{1}^{0}\right)-e^{\nu s_{2}} \mathbf{d}\left(s_{1}\right) p\left(z_{1}^{0}\right)+e^{\nu s_{2}} \mathbf{d}\left(s_{1}\right) p\left(z_{1}^{0}\right)-$ $e^{\nu s_{2}} \mathbf{d}\left(s_{2}\right) p\left(z_{1}^{0}\right)+e^{\nu s_{2}} \mathbf{d}\left(s_{2}\right) p\left(z_{1}^{0}\right)-e^{\nu s_{2}} \mathbf{d}\left(s_{2}\right) p\left(z_{2}^{0}\right) \| \leq$ $\left\|\mathbf{d}\left(s_{1}\right)\right\|_{\mathcal{L}}^{\mathbf{B}}\left\|p\left(z_{1}^{0}\right)\right\| \cdot\left|e^{\nu s_{1}}-e^{\nu s_{2}}\right|+e^{\nu s_{2}} \cdot \| \mathbf{d}\left(s_{1}\right) p\left(z_{1}^{0}\right)-$ 
$\mathbf{d}\left(s_{2}\right) p\left(z_{1}^{0}\right)\left\|+e^{\nu s_{2}}\right\| \mathbf{d}\left(s_{2}\right)\left\|_{\mathcal{L}}^{\mathbf{B}}\right\| p\left(z_{1}^{0}\right)-p\left(z_{2}^{0}\right) \|$. Since $\mathbf{d}$ is uniformly continuous then $\|\mathbf{d}(s)\|_{\mathcal{L}}^{\mathbf{B}} \leq e^{s\left\|G_{\mathbf{d}}\right\|_{\mathcal{L}}^{\mathbf{B}}}$ with $G_{\mathbf{d}} \in \mathcal{L}(B)$ and $\left\|\mathbf{d}\left(s_{1}\right) p\left(z_{1}^{0}\right)-\mathbf{d}\left(s_{2}\right) p\left(z_{1}^{0}\right)\right\|=\left\|G_{\mathbf{d}} \int_{s_{1}}^{s_{2}} \mathbf{d}(s) p\left(z_{1}^{0}\right) d s\right\| \leq$ $\sup _{z \in S}\|p(z)\| \cdot\left|e^{\mu s_{1}}-e^{\mu s_{2}}\right|$, where $\mu=\left\|G_{\mathbf{d}}\right\|_{\mathcal{L}}^{\mathbf{B}}$. The operator $p$ satisfies a Lipschitz condition on the sphere $S$, so $\left\|p\left(z_{1}^{0}\right)-p\left(z_{2}^{0}\right)\right\| \leq L_{p}\left\|z_{1}^{0}-z_{2}^{0}\right\|=L_{p} \| \mathbf{d}\left(-s_{1}\right) z_{1}-$ $\mathbf{d}\left(-s_{2}\right) z_{2}\left\|\leq L_{p}\right\|\left(\mathbf{d}\left(-s_{1}\right)-\mathbf{d}\left(-s_{2}\right)\right) z_{1}\left\|+L_{p}\right\| \mathbf{d}\left(-s_{2}\right)\left(z_{1}-\right.$ $\left.z_{2}\right)\left\|\leq L_{p}\right\| z_{1}\left\|\cdot\left|e^{\mu s_{1}}-e^{\mu s_{2}}\right|+L_{p}\right\| \mathbf{d}\left(-s_{2}\right)\left\|_{\mathcal{L}}^{\mathbf{B}}\right\| z_{1}-z_{2} \|$ for a constant $L_{p}>0$. Taking into account $s_{1}=$ $-\ln \left(\left\|z_{1}\right\|_{\mathbf{d}}\right), \quad s_{2}=-\ln \left(\left\|z_{2}\right\|_{\mathbf{d}}\right)$ we derive $\| p\left(z_{1}\right)-$ $p\left(z_{2}\right)\left\|\leq L_{1}\left|\left\|z_{1}\right\|_{\mathbf{d}}^{\nu}-\left\|z_{2}\right\|_{\mathbf{d}}^{\nu}\right|+L_{2}\left|\left\|z_{1}\right\|_{\mathbf{d}}^{\mu}-\left\|z_{2}\right\|_{\mathbf{d}}^{\mu}\right|+\right.$ $L_{3}\left\|z_{1}-z_{2}\right\|$. Hence, using Proposition 3 can be shown that $\left\|p\left(z_{1}\right)-p\left(z_{2}\right)\right\| \leq L\left\|z_{1}-z_{2}\right\|, \quad z_{1}, z_{2} \in K\left(r_{1}, r_{2}\right)$, where $L$ depends on $r_{1}, r_{2}, s_{\min }, s_{\max },\left\|G_{\mathbf{d}}\right\|$, Lipschitz constant for $p$ on the sphere $S$ and on $\sup _{z \in S}\|p(z)\|$.

II. By definition of the Fréchet derivative we have

$$
\lim _{\|\Delta h\| \rightarrow 0} \frac{\left\|p\left(z_{0}+\Delta h\right)-p\left(z_{0}\right)-\left(D p\left(z_{0}\right)\right)(\Delta h)\right\|}{\|\Delta h\|}=0
$$

for any $z_{0} \in S$. Due to $z_{0}=\mathbf{d}\left(-\ln \|z\|_{\mathbf{d}}\right) z$ and homogeneity of the operator $p$ we have $p(z)=$ $e^{\nu \ln \left(\|z\|_{\mathbf{d}}\right)} \mathbf{d}\left(\ln \left(\|z\|_{\mathbf{d}}\right)\right) p\left(z_{0}\right)$. This imminently implies the announced formula for Fréchet derivative.

\section{REFERENCES}

[1] V. I. Zubov, Methods of A.M. Lyapunov and Their Applications. Noordhoff, Leiden, 1964.

[2] W. Hahn, Stability of Motion. New York: Springer-Verlag Berlin Heidelberg, 1967.

[3] H. Hermes, "Nilpotent approximations of control systems and distributions," SIAM Journal of Control and Optimization, vol. 24, no. 4, p. 731, 1986.

[4] L. Rosier, "Homogeneous Lyapunov function for homogeneous continuous vector field," Systems \& Control Letters, vol. 19, pp. 467-473, 1992.

[5] G. Folland, "Subelliptic estimates and function spaces on nilpotent lie groups," Arkiv for Matematik, vol. 13, no. 1-2, pp. 161-207, 1975.

[6] V. V. Khomenuk, "On systems of ordinary differential equations with generalized homogenous right-hand sides," Izvestia vuzov. Mathematica., vol. 3(22), pp. 157-164, 1961 (in Russian).

[7] L. Rosier, "Etude de quelques problemes de stabilization," PhD Thesis, Ecole Normale Superieure de Cachan (France), 1993.

[8] M. Kawski, "Geometric homogeneity and stabilization," in Proc. IFAC Nonlinear Control Symposium, A. Krener and D. Mayne, Eds., Lake Tahoe, CA, 1995, pp. 164-169.

[9] A. Bacciotti and L. Rosier, Lyapunov Functions and Stability in Control Theory. Springer, 2001.

[10] S. P. Bhat and D. S. Bernstein, "Geometric homogeneity with applications to finite-time stability," Mathematics of Control, Signals and Systems, vol. 17, pp. 101-127, 2005.

[11] Y. Orlov, "Finite time stability and robust control synthesis of uncertain switched systems," SIAM Journal of Control and Optimization, vol. 43, no. 4, pp. 1253-1271, 2005.

[12] A. Levant, "Homogeneity approach to high-order sliding mode design," Automatica, vol. 41, no. 5, pp. 823-830, 2005.

[13] V. Andrieu, L. Praly, and A. Astolfi, "Homogeneous Approximation, Recursive Observer Design, and Output Feedback," SIAM Journal of Control and Optimization, vol. 47, no. 4, pp. 1814-1850, 2008.

[14] A. Polyakov, D. Efimov, E. Fridman, and W. Perruquetti, "On homogeneous distributed parameters equations," IEEE Transactions on Automatic Control, vol. (accepted), 2016.

[15] J.-M. Coron and L. Praly, "Adding an integrator for the stabilization problem," Systems \& Control Letters, vol. 17, no. 2, pp. 89-104, 1991.

[16] V. Haimo, "Finite time controllers," SIAM Journal of Control and Optimization, vol. 24, no. 4, pp. 760-770, 1986.
[17] A. Polyakov, D. Efimov, and W. Perruquetti, "Robust stabilization of mimo systems in finite/fixed time," International Journal of Robust and Nonlinear Control, vol. 26, no. 1, pp. 69-90, 2016.

[18] W. Perruquetti, T. Floquet, and E. Moulay, "Finite-time observers: application to secure communication," IEEE Transactions on Automatic Control, vol. 53, no. 1, pp. 356-360, 2008.

[19] V. Andrieu, L. Praly, and A. Astolfi, "High-gain observers with updated high-gain and homogeneous correction terms," Automatica, vol. 45, no. 2, pp. 422-428, 2009.

[20] A. Polyakov, D. Efimov, and W. Perruquetti, "Sliding mode control design for MIMO systems: Implicit Lyapunov Function approach," in European Control Conference (ECC), 2014, pp. 2612-2617.

[21] E. Ryan, "Universal stabilization of a class of nonlinear systems with homogeneous vector fields," Systems \& Control Letters, vol. 26, pp. 177-184, 1995.

[22] Y. Hong, " $\mathrm{H}_{\infty}$ control, stabilization, and input-output stability of nonlinear systems with homogeneous properties," Automatica, vol. 37, no. 7, pp. 819-829, 2001.

[23] E. Bernuau, A. Polyakov, D. Efimov, and W. Perruquetti, "Verification of ISS, iISS and IOSS properties applying weighted homogeneity," System \& Control Letters, vol. 62, no. 12, pp. 1159-1167, 2013.

[24] P. Benilan and M. Crandall, "Regularizing effects of homogeneous evolution equations," in Contributions to Analysis and Geometry, 1981.

[25] J. A. Sanders and J. P. Wang, "On the ingerability pf homogeneous scalar evolution equations," Journal of Differential Equations, vol. 147, no. 2, pp. 410-434, 1998.

[26] V. Sokolov and T. Wolf, "Classification of integrable polynomial vector evolution equations," Journal of Physics A, 2001.

[27] V. Perrollaz and L. Rosier, "Finite-time stabilization of $2 \times 2$ hyperbolic systems on tree-shaped networks," SIAM Journal of Control and Optimization, vol. 52, no. 1, pp. 143-163, 2014.

[28] J.-M. Coron, L. Hu, and G. Olive, "Stabilization and controllability of first-order integro-differential hyperbolic equations," (http://arxiv.org/abs/1511.01078), 2015.

[29] J.-M. Coron and H.-M. Nguyen, "Null controllability and finite time stabilization for the heat equations with variable coefficients in space in one dimension via backstepping approach," (https://hal.archivesouvertes.fr/hal-01228895), 2015.

[30] L. Hu and F. Di Meglio, "Finite-time backstepping boundary stabilization of $3 \times 3$ hyperbolic systems," in European Control Conference (ECC), 2015, pp. 67-73.

[31] F. Alabau-Boussouira, V. Perrollaz, and L. Rosier, "Finite-time stabilization of a network of strings," Math. Control Relat. Fields, vol. 5, no. 4, pp. 721-742, 2015.

[32] A. Pazy, Semigroups of Linear Operators and Applications to Partial Differential Equations. Springer, 1983.

[33] H. Halkin, "Implicit functions and optimization problems without continuous differentiability of the data." SIAM Journal of Control and Optimization, vol. 12, no. 2, 1974.

[34] V. A. Galaktionov and J. L. Vazquez, "Necessary and sufficient conditions for complete blow-up and extinction for one-dimensional quasilinear heat equations," Archive for Rational Mechanics and Analysis, vol. 129, no. 3, pp. 225-244, 1995.

[35] V. Korobov, Method of Controlability Functions (in Russian). NIC, Moscow, 2007.

[36] Y. Orlov, "Application of Lyapunov method in distributed systems," Automation and Remote Control, vol. 44, pp. 426-430, 1983.

[37] _ _ Discontinuous systems: Lyapunov analysis and robust synthesis under uncertainty conditions. Springer, 2008.

[38] A. Polyakov, D. Efimov, and W. Perruquetti, "Finite-time and Fixedtime Stabilization: Implicit Lyapunov Function Approach," Automat$i c a$, vol. 51, no. 1, pp. 332-340, 2015.

[39] A. Polyakov, J.-M. Coron, and L. Rosier, "Homogeneous Finite-Time Control For Evaluation Equation in Hilbert Space," IEEE Transactions on Automatic Control, 2016 (submitted).

[40] A. Polyakov and I. Chairez, "A new homogeneous quasi-continuous second order sliding mode control," in XVI Congreso Latinoamericano de Control Automatico, 2014.

[41] G. Kumagai, "An implicit function theorem: Comment," Journal of Optimization Theory and Applications, vol. 31, no. 2, pp. 285-288, 1980. 